\title{
Can a DNA Origami Structure Constrain the Position and Orientation of an
}

\section{Attached Dye Molecule?}

\author{
Divita Mathur, ${ }^{1,2}$ Young C. Kim, ${ }^{3}$ Sebastián A. Díaz, ${ }^{2}$ Paul D. Cunningham, ${ }^{4}$ Brian S. \\ Rolczynski, ${ }^{4,5}$ Mario G. Ancona, ${ }^{4}$ Igor L. Medintz, ${ }^{2}$ and Joseph S. Melinger ${ }^{4}$
}

${ }^{1}$ College of Science

George Mason University, Fairfax, VA 22030, USA

${ }^{2}$ Center for Bio/Molecular Science and Engineering, Code 6900

${ }^{3}$ Materials Science and Technology Division, Code 6300

${ }^{4}$ Electronics Science and Technology Division, Code 6800

U.S. Naval Research Laboratory, Washington DC 20375, USA.

${ }^{5}$ ASEE Postdoctoral Associate

1818 N Street N.W. Suite 600, Washington DC 20036 USA

\section{Supplementary Data}

\begin{tabular}{|c|c|c|}
\hline Content & & Page \\
\hline Fig S1 & CaDNAno diagram of the DNA bundle & $\mathrm{S}-2$ \\
\hline Table S1 & Sequences of Cy3-labeled staple strands & S-3 \\
\hline Fig S2 & Normalized absorbance and emission spectra & S-3 \\
\hline Fig S3 & Normalized fluorescence excitation spectra & S-4 \\
\hline Table S2 & Peak absorption and emission wavelengths & S-5 \\
\hline Table S3 & Amplitude and time constants of all samples & S-6 \\
\hline Table S4 & Relaxation times and $\mathrm{S}^{2}$ of all samples shown in Fig 4 & S-7 \\
\hline Fig S4 & Rigid body diffusion model & S-8 \\
\hline Fig S5 & Analysis of the different MD water models tested & S-8 \\
\hline Fig S6 & Lab- and DNA-frame MD anisotropy dynamics of $A_{d s D N A}$ and $E_{d s D N A}$ & S-9 \\
\hline Table S5 & MD relaxation times and $\mathrm{S}^{2}$ of $\mathrm{A}_{\mathrm{dsDNA}}$ and $\mathrm{E}_{\mathrm{dsDNA}}$ & S-9 \\
\hline Table S6 & Lab- and DNA-frame relaxation times and $\mathrm{S}^{2}$ of $\mathrm{A}_{\mathrm{dsDNA}}$ and $\mathrm{E}_{\mathrm{dsDNA}}$ & S-9 \\
\hline Fig S7 & $\mathrm{G}_{\text {bundle structure and MD predicted anisotropy dynamics }}$ & S-10 \\
\hline Table S7 & MD relaxation times and $\mathrm{S}^{2}$ of $\mathrm{G}_{\text {bundle }}$ & S-10 \\
\hline Fig S8 & MD snapshots of $A_{\text {bundle }}$ dynamics & S-11 \\
\hline Fig S9 & $\begin{array}{l}\text { Time-resolved Cy3 linker and dipole angle distribution in } A_{\mathrm{dsDNA}}, \\
\mathrm{G}_{\mathrm{dsDNA}}, \mathrm{A}_{\text {bundle }}, \mathrm{G}_{\text {bundle, }} \text { and } \mathrm{F}_{\text {bundle }}\end{array}$ & S-12 \\
\hline Fig S10 & $\begin{array}{l}\text { Positions and separation of } \mathrm{Cy} 3 \text { and presumed } \mathrm{Cy} 5 \text { sites in } \mathrm{A}_{\mathrm{dsDNA}} \text {, } \\
\mathrm{G}_{\mathrm{dsDNA}}, \mathrm{A}_{\text {bundle }}, \mathrm{G}_{\text {bundle, }} \text { and } \mathrm{F}_{\text {bundle }}\end{array}$ & S-13 \\
\hline Fig S11 & $\begin{array}{l}\text { Predicted FRET efficiency, separation, and } \kappa^{2} \text { in } A_{d s D N A}, G_{d s D N A}, A_{b u n d l e} \text {, } \\
G_{\text {bundle, }} \text { and } F_{\text {bundle }}\end{array}$ & S-14 \\
\hline Table S8 & Complete list of DNA bundle staple strands & S-15 \\
\hline
\end{tabular}




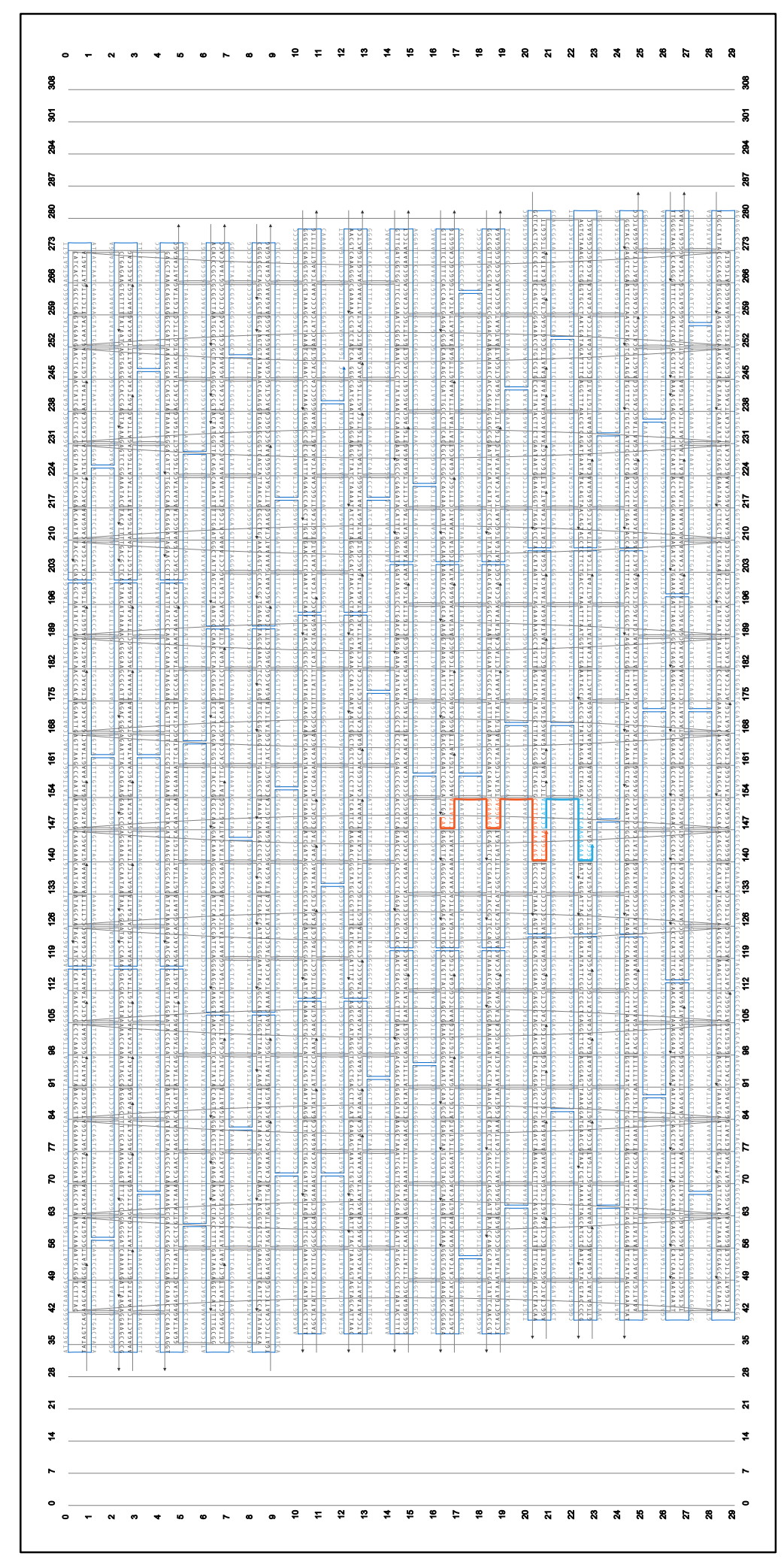

Figure S1: High resolution caDNAno diagram of the DNA bundle. Staples in Orange and Cyan represent the strands that were modified to contain Cy3. 
Table S1: List of Cy3-conjugated staple strands and their linear complementary control strands. Melting temperature calculated in the presence of $9 \mathrm{mM} \mathrm{Mg}^{2+}$.

\begin{tabular}{cllcc}
\hline Configuration & Name & Sequence 5' - 3 & Length & $\begin{array}{l}\text { Tm } \\
\left.\mathbf{(}^{\circ} \mathbf{C}\right)\end{array}$ \\
\hline A & $\begin{array}{l}\text { NT131-5'Cy3- } \\
\text { replace_base }\end{array}$ & $\begin{array}{l}\text { /5Cy3/CTATTACTCCTCAAGAGAAGGG } \\
\text { CG }\end{array}$ & 24 & 65.7 \\
\hline B & $\begin{array}{l}\text { NT118-3'Cy3- } \\
\text { replace_base }\end{array}$ & $\begin{array}{l}\text { CAGCTCATTAGGAAAGCACAGGAGCC } \\
\text { TTGAGTAACAGTTTCGGA/3Cy3sp/ }\end{array}$ & 44 & 76.0 \\
\hline C & $\begin{array}{l}\text { NT131-5'Cy3- } \\
\text { with_end_base }\end{array}$ & $\begin{array}{l}\text { /5Cy3/CCTATTACTCCTCAAGAGAAGG } \\
\text { GCG }\end{array}$ & 25 & 67.3 \\
\hline D & $\begin{array}{l}\text { NT118-3'Cy3- } \\
\text { with_end_base }\end{array}$ & $\begin{array}{l}\text { CAGCTCATTAGGAAAGCACAGGAGCC } \\
\text { TTGAGTAACAGTTTCGGAA/3Cy3sp/ }\end{array}$ & 45 & 76.0 \\
\hline E & NT131-iCy3 & $\begin{array}{l}\text { CCTATTACTCCTCA/iCy3/GAGAAGGGC } \\
\text { G }\end{array}$ & 24 & 66.9 \\
\hline F & $\begin{array}{l}\text { NT118-iCy3- } \\
\text { crossover }\end{array}$ & $\begin{array}{l}\text { CAGCTCATTAGGAAAGCACAGGAGCC } \\
\text { TTGAGTAACAGT/iCy3/TCGGAA }\end{array}$ & 44 & 76.0 \\
\hline B, D, F-comp & NT131-comp & CGCCCTTCTCTTGAGGAGTAATAGG & 25 & 67.3 \\
\hline
\end{tabular}


a

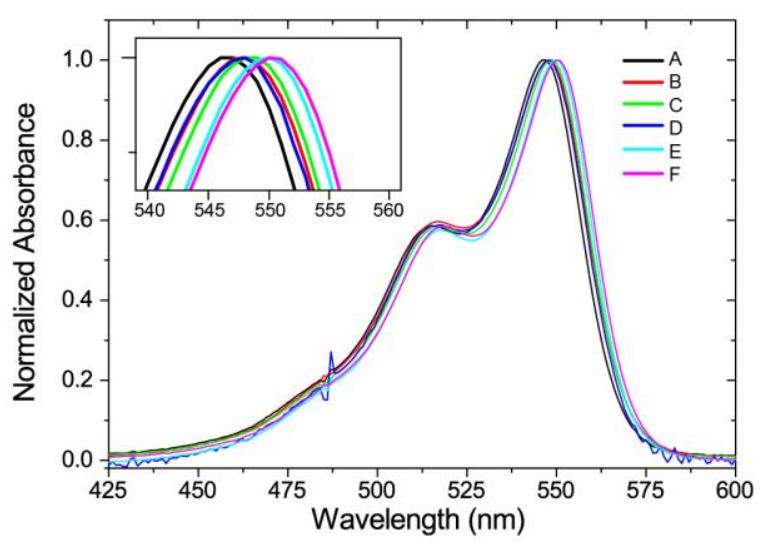

b

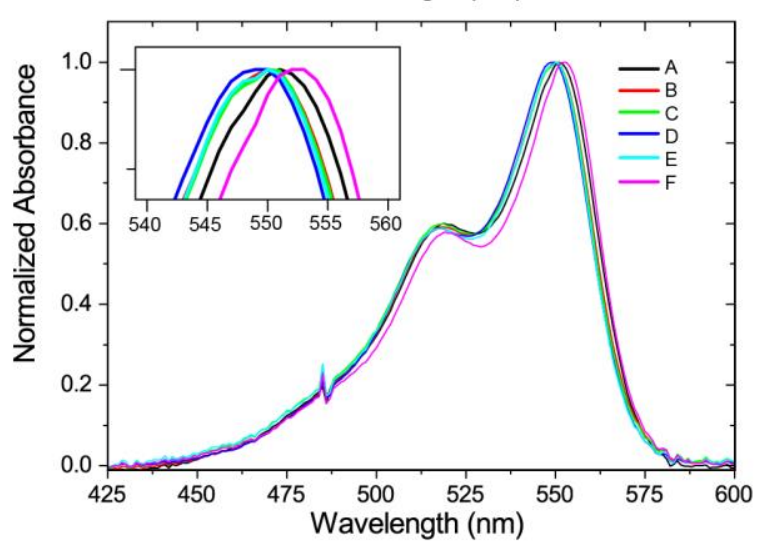

C
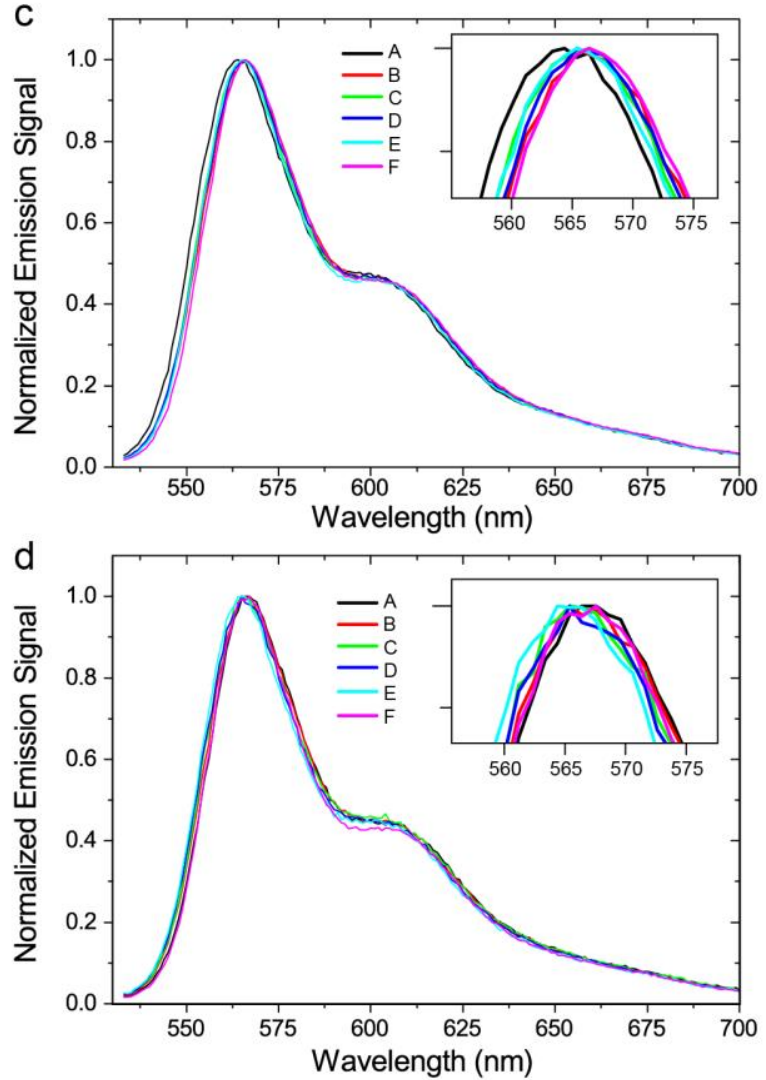

Figure S2: Steady-state spectral characterization of Cy3-conjugated DNA bundle and dsDNA. Normalized absorbance and emission spectra of $(\mathrm{a}, \mathrm{c}) \mathrm{Cy} 3$-dsDNA and $(\mathrm{b}, \mathrm{d}) \mathrm{Cy} 3$-bundle structures.
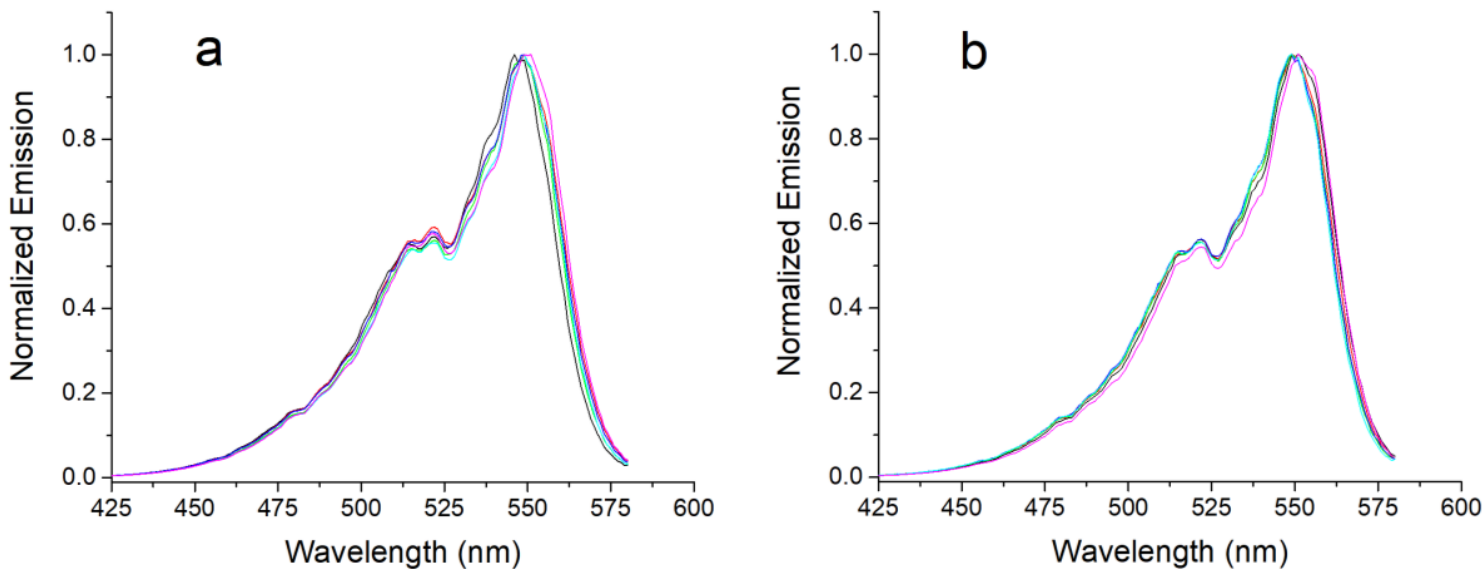

Figure S3: Normalized fluorescence excitation spectra of Cy3-dsDNA (a) and bundle (b). The legend is the same as Figure S2. 
Table S2: Comparison of peak absorption and emission wavelengths (nm) of Cy3-dsDNA and Cy3bundle.

\begin{tabular}{ccccc}
\hline & \multicolumn{2}{c}{ Absorption $\left(\lambda_{\boldsymbol{a b s}}^{\boldsymbol{m a x}}\right)$} & \multicolumn{2}{c}{ Emission $\left(\boldsymbol{\lambda}_{\boldsymbol{e m}}^{\boldsymbol{m a x}}\right)$} \\
\hline & dsDNA & Bundle & dsDNA & Bundle \\
\hline $\mathbf{A}$ & 547 & 551 & 564 & 566 \\
\hline $\mathbf{B}$ & 548 & 550 & 565 & 565 \\
\hline $\mathbf{C}$ & 549 & 550 & 566 & 565 \\
\hline $\mathbf{D}$ & 548 & 550 & 565 & 565 \\
\hline $\mathbf{E}$ & 550 & 550 & 565 & 564 \\
\hline F & 550 & 553 & 565 & 567 \\
\hline
\end{tabular}


Table S3: Amplitude and time constants derived from multi-exponential fitting of the fluorescence lifetimes.

\begin{tabular}{|c|c|c|c|c|c|c|c|c|}
\hline & & $A_{1}$ & $\tau_{1}[\mathrm{~ns}]$ & $A_{2}$ & $\tau_{2}[\mathrm{~ns}]$ & $A_{3}$ & $\tau_{3}[\mathrm{~ns}]$ & $\tau_{a v}[\mathbf{n s}]$ \\
\hline \multirow[b]{2}{*}{$\mathbf{A}$} & dsDNA & $0.35 \pm 0.02$ & $0.40 \pm 0.01$ & $0.36 \pm 0.02$ & $0.89 \pm 0.04$ & $0.05 \pm 0.002$ & $2.43 \pm 0.12$ & $0.76 \pm 0.10$ \\
\hline & Bundle & $0.47 \pm 0.01$ & $0.83 \pm 0.01$ & $0.54 \pm 0.01$ & $2.46 \pm 0.02$ & - & - & $1.70 \pm 0.08$ \\
\hline \multirow[b]{2}{*}{ B } & dsDNA & $0.39 \pm 0.02$ & $0.48 \pm 0.01$ & $0.31 \pm 0.02$ & $1.13 \pm 0.06$ & $0.11 \pm 0.002$ & $2.67 \pm 0.12$ & $1.01 \pm 0.12$ \\
\hline & Bundle & $0.54 \pm 0.01$ & $0.75 \pm 0.01$ & $0.48 \pm 0.01$ & $2.40 \pm 0.02$ & - & - & $1.55 \pm 0.07$ \\
\hline \multirow[b]{2}{*}{$\mathrm{C}$} & dsDNA & $0.34 \pm 0.02$ & $0.49 \pm 0.01$ & $0.45 \pm 0.01$ & $1.24 \pm 0.05$ & $0.03 \pm 0.002$ & $2.91 \pm 0.20$ & $0.99 \pm 0.08$ \\
\hline & Bundle & $0.60 \pm 0.01$ & $0.79 \pm 0.01$ & $0.40 \pm 0.01$ & $2.30 \pm 0.02$ & & & $1.40 \pm 0.06$ \\
\hline \multirow{2}{*}{ D } & dsDNA & $0.39 \pm 0.01$ & $0.42 \pm 0.01$ & $0.27 \pm 0.01$ & $1.12 \pm 0.05$ & $0.10 \pm 0.001$ & $2.56 \pm 0.07$ & $0.93 \pm 0.09$ \\
\hline & Bundle & $0.55 \pm 0.01$ & $0.68 \pm 0.01$ & $0.44 \pm 0.01$ & $2.21 \pm 0.02$ & - & - & $1.36 \pm 0.06$ \\
\hline \multirow{2}{*}{$\mathbf{E}$} & dsDNA & $0.49 \pm 0.01$ & $0.82 \pm 0.01$ & $0.42 \pm 0.01$ & $1.90 \pm 0.02$ & - & - & $1.30 \pm 0.06$ \\
\hline & Bundle & $0.59 \pm 0.01$ & $0.97 \pm 0.01$ & $0.44 \pm 0.01$ & $2.35 \pm 0.02$ & - & - & $1.50 \pm 0.06$ \\
\hline \multirow[b]{2}{*}{$\mathbf{F}$} & dsDNA & $0.51 \pm 0.01$ & $0.86 \pm 0.01$ & $0.40 \pm 0.01$ & $2.23 \pm 0.02$ & - & - & $1.45 \pm 0.07$ \\
\hline & Bundle & $035 \pm 0.01$ & $0.79 \pm 0.01$ & $0.76 \pm 0.01$ & $2.70 \pm 0.02$ & - & - & $2.25 \pm 0.10$ \\
\hline
\end{tabular}


Table S4: Summary of the short $\left(\tau_{e}\right)$ and long $\left(\tau_{M}\right)$ anisotropy decay constants and restriction constant $\left(S^{2}\right)$ derived from the bi-exponential fitting (Eq. 3) of fluorescence anisotropy dynamics.

\begin{tabular}{|c|c|c|c|c|c|}
\hline Case & Description & & $\boldsymbol{\tau}_{\boldsymbol{e}}[\mathrm{ns}]$ & $\tau_{M}[\mathrm{~ns}]$ & $S^{2}$ \\
\hline \multirow{2}{*}{ A } & \multirow{2}{*}{5 '-end replacing base } & dsDNA & $0.70 \pm 0.05$ & $10.3 \pm 0.2$ & $0.79 \pm 0.01$ \\
\hline & & Bundle & $0.86 \pm 0.07$ & $70 \pm 4$ & $0.87 \pm 0.01$ \\
\hline \multirow{2}{*}{ B } & \multirow{2}{*}{ 3'-end replacing base } & dsDNA & $0.42 \pm 0.03$ & $15.9 \pm 0.3$ & $0.76 \pm 0.01$ \\
\hline & & Bundle & $0.63 \pm 0.08$ & $84 \pm 5$ & $0.87 \pm 0.01$ \\
\hline \multirow{2}{*}{$\mathrm{C}$} & \multirow{2}{*}{$\begin{array}{l}\text { 5'-end coupled to last } \\
\text { base }\end{array}$} & dsDNA & $0.62 \pm 0.04$ & $11.5 \pm 0.2$ & $0.91 \pm 0.01$ \\
\hline & & Bundle & $0.67 \pm 0.13$ & $71 \pm 4$ & $0.87 \pm 0.01$ \\
\hline \multirow{2}{*}{$\mathrm{D}$} & \multirow{2}{*}{$\begin{array}{l}\text { 3'-end coupled to last } \\
\text { base }\end{array}$} & dsDNA & $0.49 \pm 0.04$ & $20.6 \pm 0.2$ & $0.76 \pm 0.01$ \\
\hline & & Bundle & $0.50 \pm 0.06$ & $46 \pm 3$ & $0.87 \pm 0.01$ \\
\hline \multirow{2}{*}{$\mathrm{E}$} & \multirow{2}{*}{$\begin{array}{l}\text { Internal away from } \\
\text { crossovers }\end{array}$} & dsDNA & $1.1 \pm 0.1$ & $15.4 \pm 0.5$ & $0.83 \pm 0.01$ \\
\hline & & Bundle & $0.76 \pm 0.10$ & $79 \pm 5$ & $0.88 \pm 0.01$ \\
\hline \multirow{2}{*}{$\mathrm{F}$} & \multirow{2}{*}{$\begin{array}{l}\text { Internal at crossover } \\
\text { site }\end{array}$} & dsDNA & $1.0 \pm 0.05$ & $16.8 \pm 0.5$ & $0.81 \pm 0.01$ \\
\hline & & Bundle & $1.30 \pm 0.13$ & $127 \pm 10$ & $0.91 \pm 0.01$ \\
\hline
\end{tabular}




\section{Rigid body diffusion based on ellipsoid model}

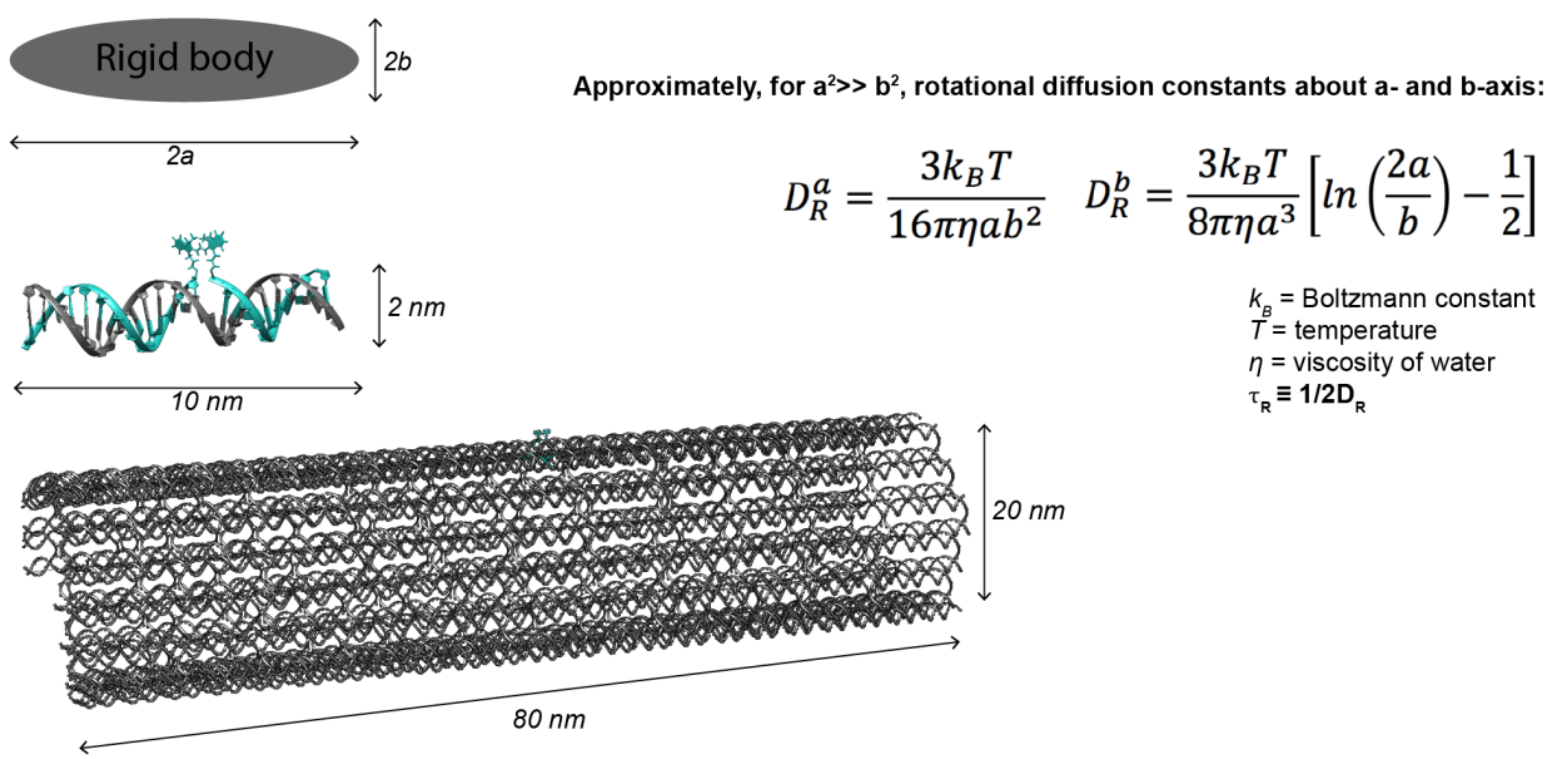

Figure S4: Rigid body diffusion analysis of dsDNA and DNA bundle.

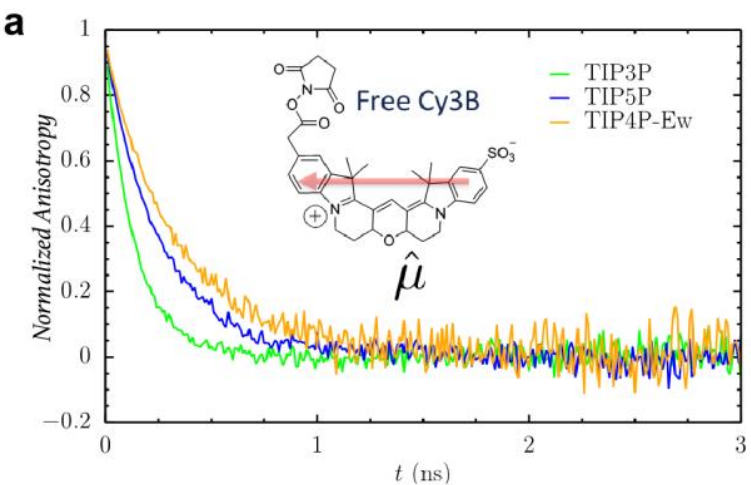

\begin{tabular}{l|c|c} 
b & $\begin{array}{c}\text { Diffusion constant } \\
\left(10^{-5} \mathrm{~cm}^{2} / \mathrm{s}\right)\end{array}$ & $\begin{array}{c}\text { Relaxation time } \\
\text { for Cy3B }(\mathrm{ns})\end{array}$ \\
\hline TIP3P & 5.19 & 0.13 \\
\hline TIP4P-Ew & 2.4 & 0.33 \\
\hline TIP5P & 2.62 & 0.26 \\
\hline Experiment & 2.30 & 0.38
\end{tabular}

Figure S5: Analysis of different MD water models for dye anisotropy dynamics simulation in comparison to the experimental values. 


\section{A-dsDNA}
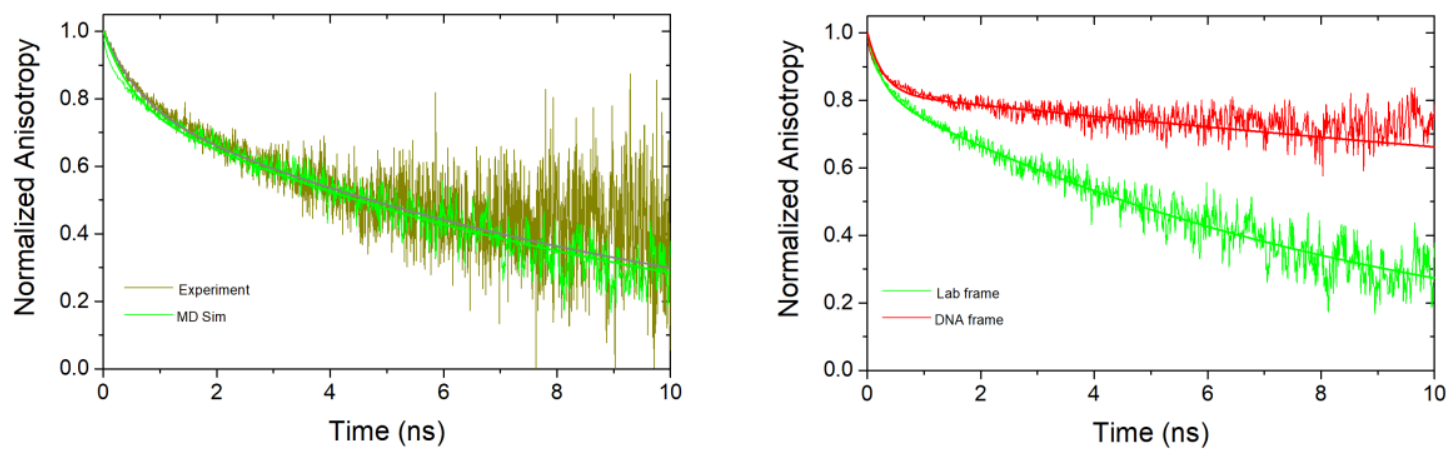

\section{E-dsDNA}
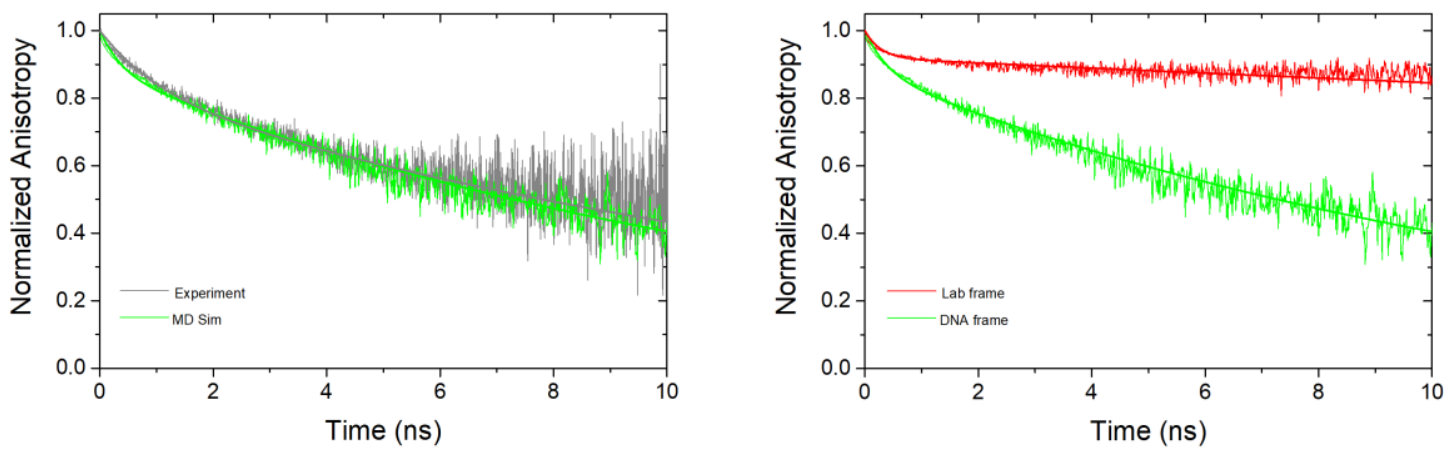

Figure S6: MD and experimental anisotropy dynamics (left), and lab-frame and DNA-frame anisotropy (Right) of $\mathrm{A}_{\mathrm{dsDNA}}$ and $\mathrm{E}_{\mathrm{dsDNA}}$. Solid curves are fits to the data using Eq 3 in the main text.

Table S5: MD relaxation times and $S^{2}$ derived by bi-exponential fitting for $\mathrm{A}_{\mathrm{dsDNA}}$ and $\mathrm{E}_{\mathrm{dsDNA}}$.

\begin{tabular}{ccccc}
\hline & & $\boldsymbol{\tau}_{\boldsymbol{e}}[\mathbf{n s}]$ & $\boldsymbol{\tau}_{\boldsymbol{M}}[\mathbf{n s}]$ & $\boldsymbol{S}^{\mathbf{2}}$ \\
\hline $\mathrm{A}$ & $\mathrm{dsDNA}$ & 0.25 & 9.0 & 0.83 \\
\hline $\mathrm{E}$ & $\mathrm{dsDNA}$ & 0.32 & 11.5 & 0.90 \\
\hline
\end{tabular}

Table S6: Comparison of relaxation times and $S^{2}$ for $\mathrm{A}_{\mathrm{ds} D N A}$ and $\mathrm{E}_{\mathrm{dsDNA}}$ in Lab- and DNA-frames.

\begin{tabular}{ccccc}
\hline & $\mathbf{A}_{\text {dsDNA }}$ Lab-frame & A dsDNA DNA-frame $_{\text {ds }}$ & E $_{\text {dsDNA }}$ Lab-frame & E $_{\text {dsDNA }}$ DNA-frame \\
\hline$S^{2}$ & 0.83 & 0.82 & 0.90 & 0.92 \\
\hline$\tau_{e}$ & $0.25 \mathrm{~ns}$ & $0.28 \mathrm{~ns}$ & $0.32 \mathrm{~ns}$ & $0.27 \mathrm{~ns}$ \\
\hline$\tau_{M}$ & $9.0 \mathrm{~ns}$ & $46.7 \mathrm{~ns}$ & $11.5 \mathrm{~ns}$ & $118.7 \mathrm{~ns}$ \\
\hline
\end{tabular}



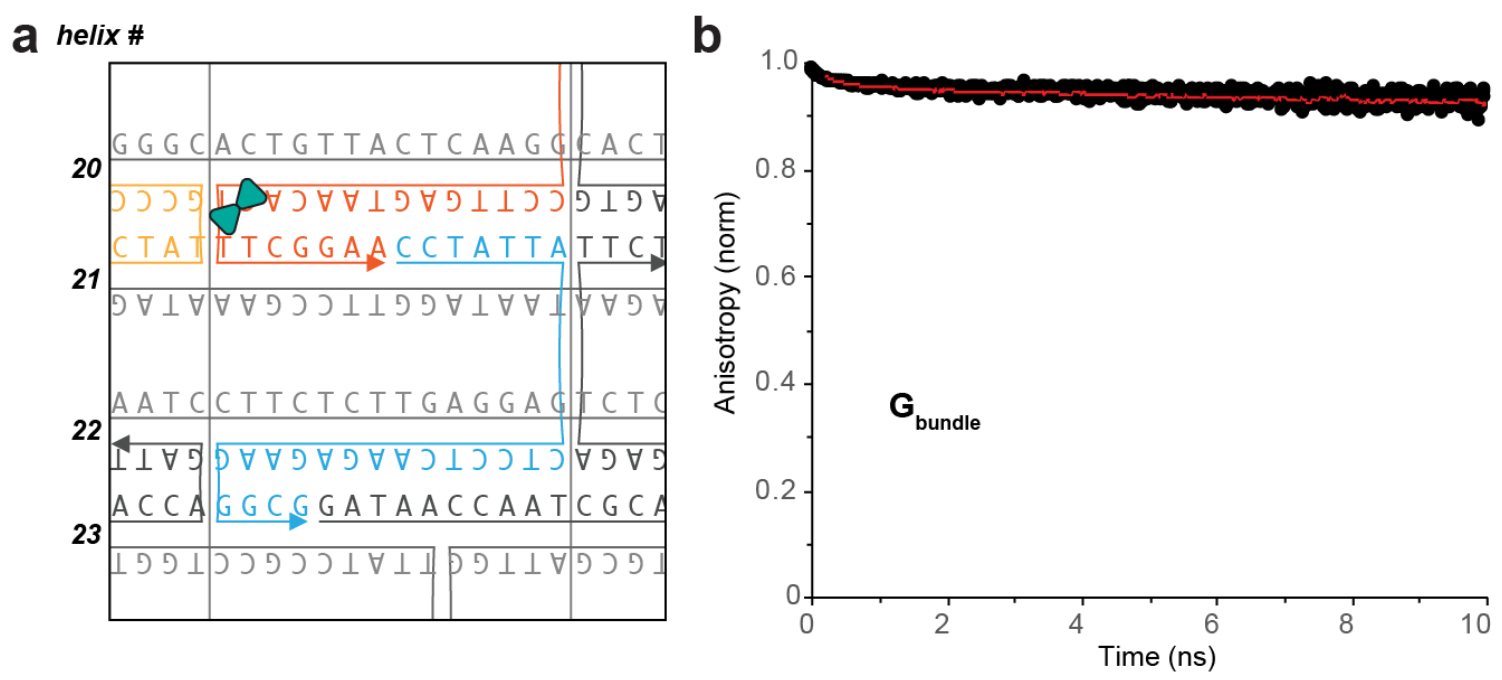

Figure S7: $\mathrm{G}_{\text {bundle }}$ structure and MD predicted anisotropy.

Table S7: Predicted relaxation times and $\mathrm{S}^{2}$ derived by bi-exponential fitting for $\mathrm{G}_{\text {bundle. }}$.

\begin{tabular}{ccccc}
\hline & & $\boldsymbol{\tau}_{\boldsymbol{e}}[\mathbf{n s}]$ & $\boldsymbol{\tau}_{\boldsymbol{M}}[\mathbf{n s}]$ & $\boldsymbol{S}^{\mathbf{2}}$ \\
\hline $\mathrm{G}$ & Bundle & 0.33 & 345 & 0.97 \\
\hline
\end{tabular}




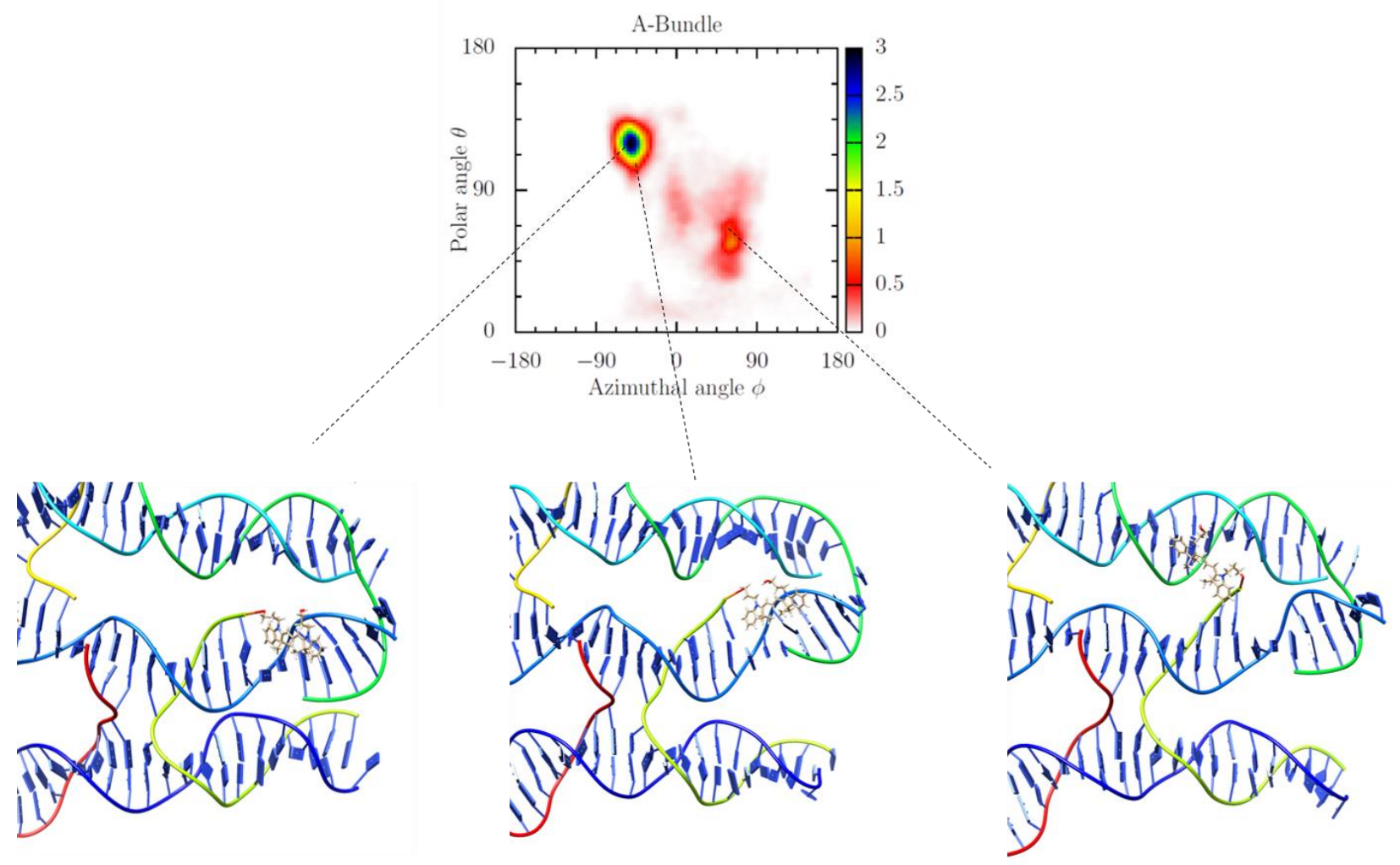

Figure S8: MD snapshots of $A_{\text {bundle }}$ taken from three parts of the color map, as indicated by the lines. The snapshots from the blue and orange regions show that $\mathrm{Cy} 3$ aligns with the DNA groove. The snapshot from the red regions shows $\mathrm{Cy} 3$ outside the groove region. 


\section{Cy3 Dipole Angles}
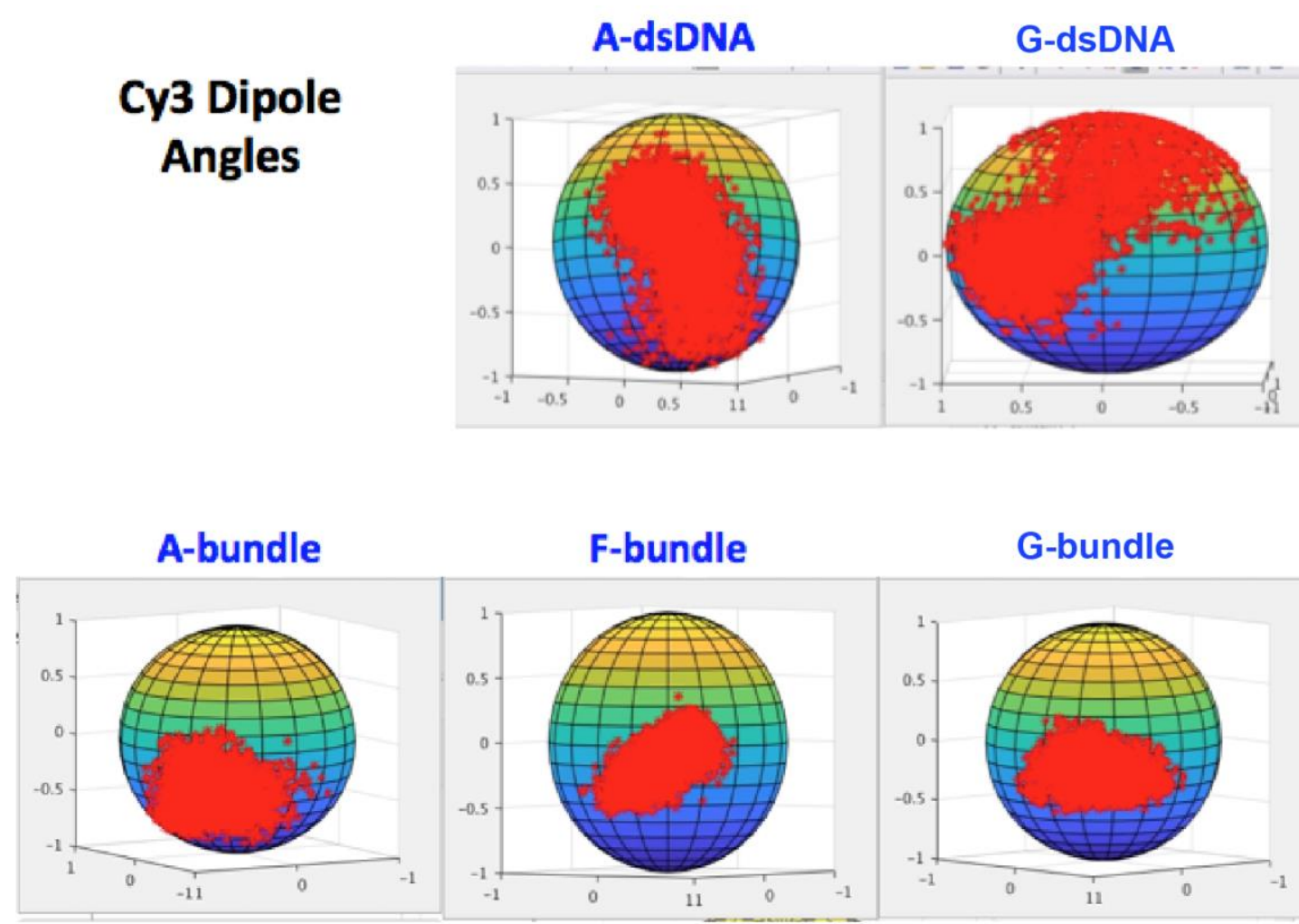

Cy3 Linker Angles
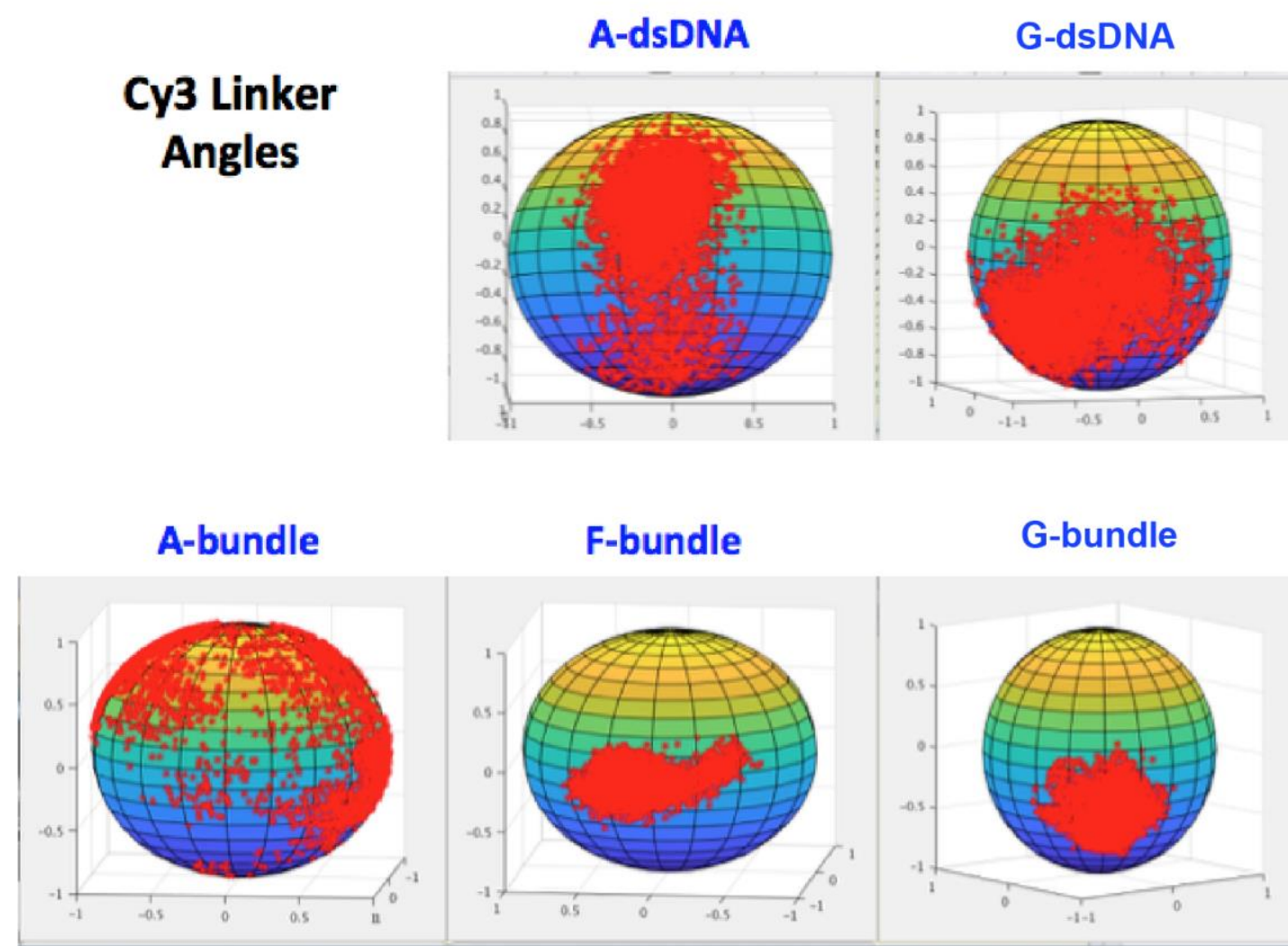

Figure S9: Cy3 dipole and linker angles used in FRET simulations. The spacing for latitude and longitude lines is 15 degrees and 20 degrees, respectively. 


\begin{tabular}{|lcccc|}
\hline Sites & $\mathbf{1}$ & $\mathbf{2}$ & $\mathbf{3}$ & $\mathbf{4}$ \\
\hline $\mathrm{A}_{\text {dsDNA }}$ & 30.84 & - & - & - \\
$\mathrm{G}_{\text {dsDNA }}$ & 30.84 & - & - & - \\
$\mathrm{A}_{\text {bundle }}$ & 27.16 & 30.06 & 29.57 & 29.57 \\
$\mathrm{~F}_{\text {bundle }}$ & 30.21 & 30.00 & 29.16 & 29.86 \\
$\mathrm{G}_{\text {bundle }}$ & 29.59 & 30.86 & 31.96 & 29.91 \\
\hline
\end{tabular}
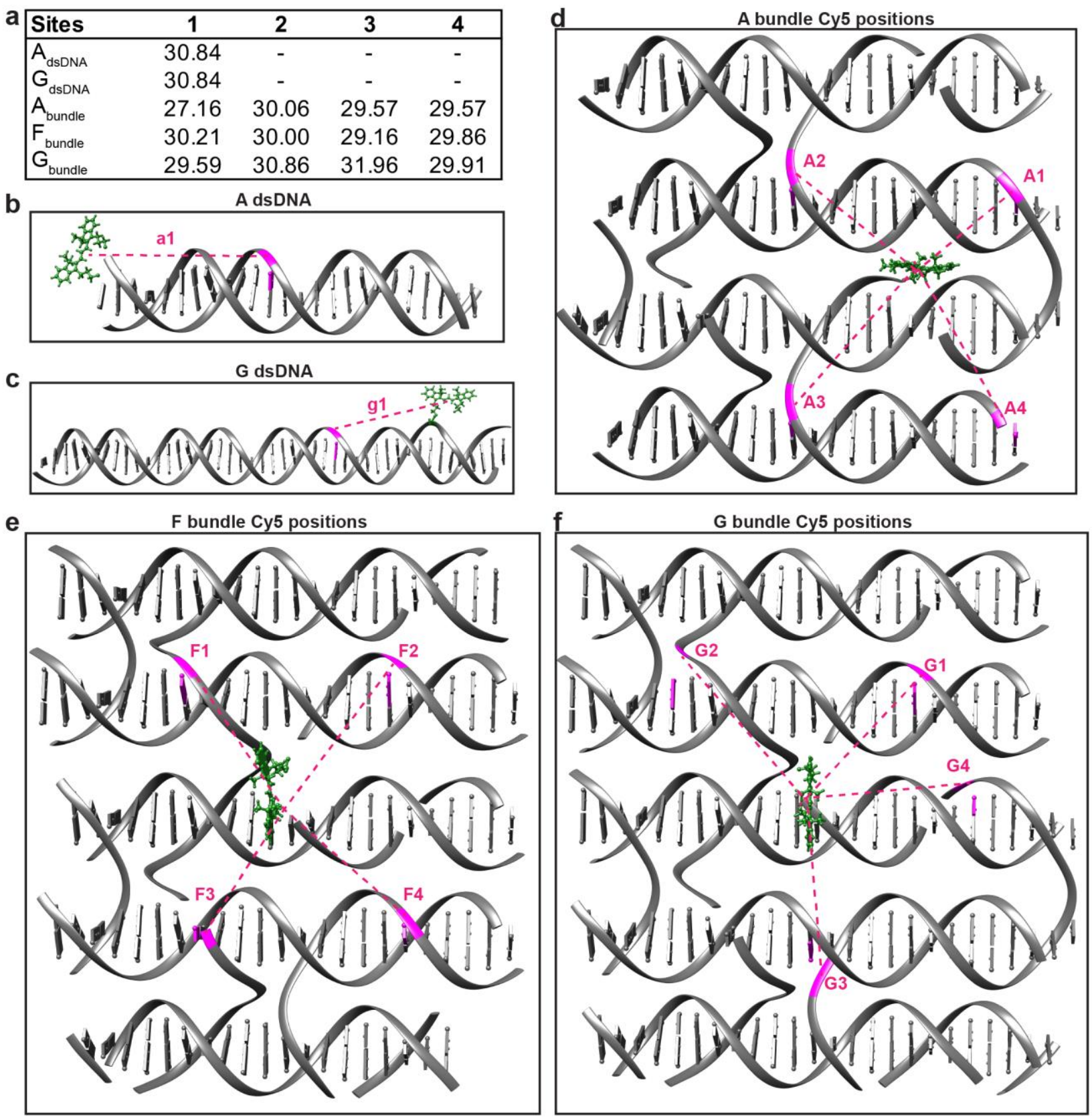

Figure S10: (a) Separation of Cy5 sites from Cy3 in each configuration (angstroms $\AA$ ). (b-f) Positions of presumed Cy5 sites (pink) relative to $\mathrm{Cy} 3$ (green) on $\mathrm{A}_{\mathrm{dsDNA}}, \mathrm{G}_{\mathrm{dsDNA}}, \mathrm{A}_{\text {bundle }}, \mathrm{F}_{\text {bundle }}$ and $\mathrm{G}_{\text {bundle. }}$. 

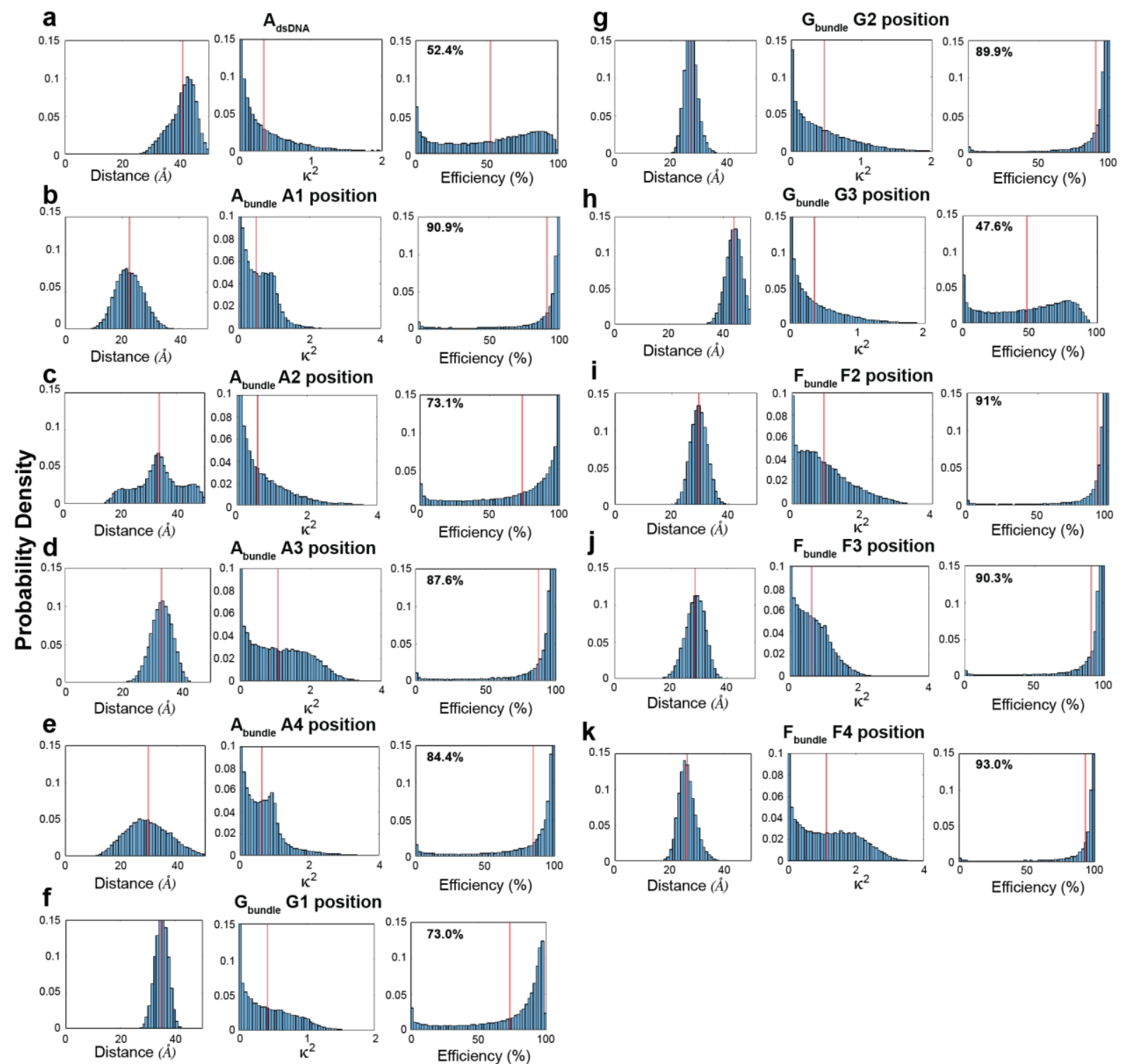

Figure S11: Predicted probability densities of inter-dye distance $(\AA)$, Orientation $\left(\kappa^{2}\right)$, and FRET Efficiency (\%) at sites selected in different configurations. The vertical red lines indicate the weighted average for each quantity. 
Table S8: Complete list of staple strands used for DNA bundle formation. Staples NT118 and NT135 were accordingly replaced by $\mathrm{Cy} 3$-modified staple strands.

\begin{tabular}{|c|c|c|}
\hline Name & Sequence (5'-3') & $\operatorname{Tm}\left({ }^{\circ} \mathrm{C}\right)$ \\
\hline NT001 & TATCTTCATTGAATCCCATGGGCG & 57 \\
\hline NT002 & TTTTTATAGTCAGGGAAGCCCGTTTT & 57 \\
\hline NT003 & ATTGCGGTAAGAGGGATCAAAAAGGCGTTTT & 62 \\
\hline NT004 & GACTGGATTTGCAAAAGAAGTAATTACG & 55 \\
\hline NT005 & GCGGAATAAACCAAAATAGCGCAACACT & 60 \\
\hline NT006 & AGAAAGCAAATCTTTACGTCGGATTCTCCGTGTGAGCG & 66 \\
\hline NT007 & CCATAGTAAAGACCATAAACGGCGGATTGACGTATG & 62 \\
\hline NT008 & CCAAAAGACGACGATAACGTCA & 55 \\
\hline NT009 & TTTTAAAGACTTCCCAACAGGTTTTT & 54 \\
\hline NT010 & AATTCGAGAACCAGACCGGAACTTTAATGATAAGAGCTG & 63 \\
\hline NT011 & AGGCATAATACATAACGCCAAACGAACTGGG & 62 \\
\hline NT012 & GAGAGAGGCTTAGCGTCAACAGTTGGATAGGTCACGTTACGTTAG & 66 \\
\hline NT013 & ATCATAATACCACATTCAACTATTACAGCATTATACCTTATGTTTC & 60 \\
\hline NT014 & ACGCAGAGATTTAGGAACCCTC & 57 \\
\hline NT015 & TTTGCGGGGATTAGAGAGTACGCAAACTAAATATCATTA & 61 \\
\hline NT016 & TCTACCTTTTTGCTCGTTAATAAAAAAAAGCGCTTCGGTTTG & 63 \\
\hline NT017 & AAGACAACATGGCAACTACCAGAAACACCAGA & 63 \\
\hline NT018 & GGACGTTAACGGAACAACATTAATGCAGGTAA & 60 \\
\hline NT019 & AAGGTGAACTGGCTGTAGAAAGATTC & 57 \\
\hline NT020 & AATGAAGTTTGAACGAGAAATGGTCAATAACCTGTTTTTT & 61 \\
\hline NT021 & TATAACACTTAGAGCTTAATTGGTCATT & 53 \\
\hline NT022 & ACGGTGTCTGATAATGCTGTAGCTAAAA & 58 \\
\hline NT023 & AACGTAAATTTTCAGTGAATAAGGGATATTC & 54 \\
\hline NT024 & GCCAGATGGTTTAACGAT & 50 \\
\hline NT025 & TTTTTGATTCCCAATTCTGCCATTCCA & 58 \\
\hline NT026 & ACGAGTATTTAATCATAATATTTTTATGTGAATTACCAGTCA & 57 \\
\hline NT027 & ATACGCTGAAAAGTGACAAATCTGTGGC & 60 \\
\hline NT028 & ATCACAAAGCTGCTCAGGGC & 58 \\
\hline NT029 & TTTTTAGCTATATTTTCATTTGGTAATAGTAGTAGCATTAACTTTT & 58 \\
\hline NT030 & ATTACCCTGGCTGACCTTCATATAAAGC & 58 \\
\hline NT031 & TACGGCGCGAATTTCGCTAGATTTAGTTTGAAAGT & 62 \\
\hline NT032 & ATCAAAGAATTAGCAAAAATCGGTTGTACCAAAGGATAAGTAA & 61 \\
\hline NT033 & CGGTAGGCGCATAGGCAAAT & 58 \\
\hline NT034 & TTTTATCCAATAAATCATACAGGATGACCCTGTAATACTTTTTTTT & 60 \\
\hline NT035 & GCACAAGAGTAGAACCGCTTGCCCTGACGATTAG & 65 \\
\hline NT036 & CTTGAACACAGACAGAGCATACTCATATCAAT & 58 \\
\hline NT037 & AGGGGTGTACAGACCCATAGCCCC & 64 \\
\hline NT038 & CCACCACCGAACTGACCAACTTTGCAATCATTTAGCCGAAATCCGAAGA & 69 \\
\hline
\end{tabular}




\begin{tabular}{|c|c|c|}
\hline NT039 & TTTTGCGGGAGAAGCCAAAGGCCGGAGATTTT & 67 \\
\hline NT040 & GGGTGAGTTTATTTCAACGCAAAACATTCAAGGCAATTC & 63 \\
\hline NT041 & AGAAGTACAAAGCGATTGGTGAGGTTTCAGTAGCTATTGCCTGAG & 66 \\
\hline NT042 & TGTGTAGAAATTTTTAGAACCAAGCTAATTAA & 54 \\
\hline NT043 & GCCATCGCCTAAACACTCGGGTAAGGCTTTGAGGACTAAAGAGAA & 68 \\
\hline NT044 & GATTGGCCATGTTACAAGG & 51 \\
\hline NT045 & TTTTCAGTCAAATCACATATTCAACCGTTTTT & 57 \\
\hline NT046 & TGTGTCGGAACGAGGCAAATGATTTTGCAGACGGTAAAG & 66 \\
\hline NT047 & AAGCATATGCATCAGCGAAACAATTCAAAA & 58 \\
\hline NT048 & GACCCCCCGGAGATTTGTATCTGAGTAA & 61 \\
\hline NT049 & GGCCTACGAAGAGGGTAGCAACGGGGATCGT & 68 \\
\hline NT050 & TCCAGTCTAAAACGACGAC & 52 \\
\hline NT051 & TTTTTCTAGCTGATAAGATCTACAATTTT & 52 \\
\hline NT052 & AGTTCAGCATCGGAACGGCA & 60 \\
\hline NT053 & TTTTAGGCTATCATGTACCCCGTTTT & 57 \\
\hline NT054 & AGTCTGGAAAACTAGCATGTCGCCCC & 63 \\
\hline NT055 & TCGCCGCTAAAGGATGAACGGACCGATA & 64 \\
\hline NT056 & GCGCTACAGAAATACGTAATGCCAAAAAGAATACACTAGATAAAT & 62 \\
\hline NT057 & CACCCTCTCGCTGAGGCTTGCGACA & 67 \\
\hline NT058 & ATAGGTCATTTTTGAGAATTAATGCCGGAGAATACC & 60 \\
\hline NT059 & CGTAGCAAACAAGACTTAAGTTTCCATTAAACATCTTT & 59 \\
\hline NT060 & CGGGCGATATATTCGGAGCA & 57 \\
\hline NT061 & TTTTGTTGATAATAGCAAATATTTTT & 47 \\
\hline NT062 & AAAAAAACATTCTTACAGGAAGAAATATTTGGAACGC & 59 \\
\hline NT063 & GTTGCGCCAGCTTGCTTTCGATTAAATTCAGCTCA & 65 \\
\hline NT064 & ACAACCATCGCCTTTAATTGTATCCACGTTGAAGGAAT & 63 \\
\hline NT065 & TAAGCTCCAAAAGGAGCCCAC & 57 \\
\hline NT066 & AATAATTAAATTGTAAACGTTTTGTATACAGAAAAAATC & 54 \\
\hline NT067 & ACCAATATGTTAAAATTCGCAGGTGAATGCTTGATTAAT & 60 \\
\hline NT068 & АATAAATTTTGTTAATTTTTTGGTTTATCGACAATAGGGAGTTTTT & 59 \\
\hline NT069 & ССTCATTTTCAGGAACAACTAAAAATCTCCAAA & 58 \\
\hline NT070 & AGCCAGCATTAAATGGGAACAAATCAAAAATCAGGGCGG & 66 \\
\hline NT071 & CAACTTTATTTTCTCGTAATGCAGAAAACGAGAATATGTTTA & 59 \\
\hline NT072 & GAGTGAGTTTCCAGGGTGTAGCCTCAAATGCTTTACAATACT & 65 \\
\hline NT073 & AGTAACATCTGGCCTTCCTGTCATCAAA & 60 \\
\hline NT074 & GGATTCAACTTTCATTTGCTAAATTTTTTA & 53 \\
\hline NT075 & TAAATGACAACAGTTTCAGCGTGCGAAT & 59 \\
\hline NT076 & CATCGTAAAAGTTTTGTCGTCAATA & 52 \\
\hline NT077 & CCAGCGCTAATATCAGTCCGGCACCGCTTCTCGTCGC & 70 \\
\hline NT078 & TAAATATTACCGAAGCATAC & 45 \\
\hline NT079 & GTAAGCAAGGAAACGCAATAAAGACTCC & 58 \\
\hline
\end{tabular}




\begin{tabular}{|c|c|c|}
\hline NT080 & AAAGTTAACTAGAATTACCAGAAGGTTA & 52 \\
\hline NT081 & AAGTCAGCAGGGAAGCGCATTTGAAAAT & 61 \\
\hline NT082 & GGACCTTTTTATAGCAAATCTGCCAGTTTGATCATAGT & 61 \\
\hline NT083 & CCGGATAGCCATAAGAGACGACAGTATCGGCGTAGCAT & 67 \\
\hline NT084 & GGGGAACACCAGAATTGAGATCGC & 60 \\
\hline NT085 & CTCAATAACATAAAAAAGGGTAA & 47 \\
\hline NT086 & GTTTACCAGAACTGGCAGAA & 53 \\
\hline NT087 & TTATTACATAAAGGTGGCAACAGTTTATGGGCGACAGGTGAACACC & 66 \\
\hline NT088 & GCAAATAACGTTGTTCGTAGAAAATAGAAAATTACCAGTATT & 59 \\
\hline NT089 & AGCAGCCATCCAAATAAGAAATGCCAGTACGAGCGTTTATCCTCCC & 68 \\
\hline NT090 & CCСTTTTATTTATCCCATTTACAG & 50 \\
\hline NT091 & ATCAGTTAAGACACCACGGAATAATATAAAATGATTATAAC & 57 \\
\hline NT092 & GACAAAATTTGTCACAATCAATACATACGCAGTATGAAA & 59 \\
\hline NT093 & TCCAGATGGTTTCATAGCCTAATTCGATTTTTCAAAAAAGAC & 61 \\
\hline NT094 & AATGCCCAACGCTATACAAAATAAACAG & 56 \\
\hline NT095 & TTTAAAAATATTGACGGAATTAGA & 47 \\
\hline NT096 & TTGGAAGCCTCCAGGCTATAGAAATGAAACCCGCACTC & 67 \\
\hline NT097 & ATTTGGGAAATTATTCATTAAATTCAACCGATTGAGGGAGGG & 62 \\
\hline NT098 & GACTTAGCAAGCAGCACCGTAATCCAGA & 62 \\
\hline NT099 & CAAGACCGTTTATCATTAGTTGCCGCCAAA & 61 \\
\hline NT100 & AGGTTTTCACCCAGCTACAATTCTT & 57 \\
\hline NT101 & GACAGAACGCCCAATAGCAAGCAAAGCCGTTTTTATTTCCAATCAATA & 66 \\
\hline NT102 & TGAGAGTAGCGAACCTGAAT & 53 \\
\hline NT103 & TTGAGCAAAATCACCAGACAGA & 55 \\
\hline NT104 & ACCGCGCGAGGCGTTTCCAGC & 68 \\
\hline NT105 & CAACGTAAAGTTTGCCTATTTT & 50 \\
\hline NT106 & CTGTATAAACGGTATGCGCGTTTTGCGTTTGCCATCTTCACCCTCAGAG & 69 \\
\hline NT107 & TACATCGATAGGCCGGAAACGTCATAAAT & 59 \\
\hline NT108 & ATCGAGATTCCTTATCATTCCTCAAAAT & 55 \\
\hline NT109 & ATCGCCACCACCGTCCCATAATAGAACCGCCTGTTCAGCTCCA & 71 \\
\hline NT110 & GTAGAAATCATCGTAGGAAACCC & 53 \\
\hline NT111 & CTTATTACATCGGCTTAGCGTAGTAGCGTAGCACCATTACCATTGAGCC & 67 \\
\hline NT112 & CACCGGAAGCCGCCACCCTCACAGAGCCTTGA & 72 \\
\hline NT113 & GAGGCTGTCTACAAGCAATCAGATTATCCGGTATTCTATTGCGGG & 66 \\
\hline NT114 & AAATCCTAATTTACGATAGATTAG & 46 \\
\hline NT115 & AGGATTAGATAAGTCCTGAACACCTGTTTACAA & 59 \\
\hline NT116 & GAACCCTCAGAGCCGCCTCAGAC & 64 \\
\hline NT117 & AGGCAGGACCAGAACCACCACGAACCGCTTCATAAAAGAACGCAAG & 70 \\
\hline NT118 & CAGCTCATTAGGAAAGCACAGGAGCCTTGAGTAACAGTTTCGGAA & 67 \\
\hline NT119 & GACAGGCAGAATTTAACGTGTTATTATGCTTTAACGGGACAT & 63 \\
\hline NT120 & AAGAGTAATAGGCTTAAGTATAAAGAAAAAGCCTGTTTGCGTTAA & 62 \\
\hline
\end{tabular}




\begin{tabular}{|c|c|c|}
\hline NT121 & GACAACAAAGTACCGATCAA & 50 \\
\hline NT122 & CTGCTCCTTGATATTCATACCGT & 54 \\
\hline NT123 & ATTTGACGACAATCAGCAGCCGCAAACAACATCCCTCAGACCA & 69 \\
\hline NT124 & TCGAGCCGTAAAGTAATTCTGTAATGCAGAACGCGAGAA & 65 \\
\hline NT125 & CTGAATTCAAACAAATAAATCGAGGTTG & 54 \\
\hline NT126 & TTATCACAACAGTAGAGAGA & 47 \\
\hline NT127 & CCAACAAGCGTCATACATAAAC & 52 \\
\hline NT128 & CTTACCATTGAGAATCGCCATGGCATTT & 60 \\
\hline NT129 & CCTATCATAATTACTAGCCAA & 47 \\
\hline NT130 & GCGAAAGAAATGCCCCCATTAG & 57 \\
\hline NT131 & CCTATTACTCCTCAAGAGAAGGGCG & 58 \\
\hline NT132 & GAAGTCAGTGTGTACTGGTAATAAAACGCCAACAGAATAAGCCATGTA & 65 \\
\hline NT133 & GAAAGGTGATAAATAAGAGTATCAACAAATT & 53 \\
\hline NT134 & ATAAGAAATTTAATGGTTTGATTCAAATATATTTTCCGGCTT & 58 \\
\hline NT135 & AGGTGCCTATGCCCGTATGGCTTTTGATGATGCAGTCT & 68 \\
\hline NT136 & ACCGTTATTAAGAGCAAAGAACGCGAGAAACTATA & 61 \\
\hline NT137 & ACCTCTTCTGACCTAATAAACAC & 52 \\
\hline NT138 & GCATAACGTTTTGCTCTGATA & 51 \\
\hline NT139 & GATAACCAATCGCAAGAGCTGAGATTCT & 59 \\
\hline NT140 & TGCAAATGTGCCGTCGTGTATCACGCCACC & 67 \\
\hline NT141 & TGTAAATTTTAGTACCGCCAGTGAATTTGTTATATAAACTTTAATACCG & 61 \\
\hline NT142 & AGGTTGGATCAAAAGCTGAGA & 54 \\
\hline NT143 & АСCACСTTTTTAACCTAGTTAAT & 50 \\
\hline NT144 & AAAAAGGTATAGCCCGGAATAGGAGAGGGTAGTACCAGATT & 65 \\
\hline NT145 & TCAGAACCCGTACTCAGGAGGGCTGA & 64 \\
\hline NT146 & CAGAACCTCACCAGAACGCCTCTCAGGAAGTTAAGCCCAATAGAAC & 68 \\
\hline NT147 & GAAACGATTAAGACTCATAGGTCTGAGA & 56 \\
\hline NT148 & GAAAGGGATAGCAAGCCACGATCTACCGTGCTAGC & 66 \\
\hline NT149 & GTACCGTACAGCCCGGGGACGCAAGAAACAATGAAAAGAAAA & 67 \\
\hline NT150 & ACATAGCTAATTTTCCAGCTTAGAGATAACCCACACTGAACA & 63 \\
\hline NT151 & TAGCGTACAATAGGAACCCATCTCAGAGCCACCAC & 65 \\
\hline NT152 & TCCACAGAACACTGAGTTTCGGCCACCC & 66 \\
\hline NT153 & TATTAATGATAGCTTAAAACA & 42 \\
\hline NT154 & ACTCCAGCCCTTAGAAACTACTACAATCCTTGAAAAGAGTCAATACCCT & 66 \\
\hline NT155 & TTGAGCCATTGCAATGACG & 54 \\
\hline NT156 & ATGTCCAAGTCTGGAAATACCTATTTACATTGGCAGACATTC & 63 \\
\hline NT157 & CGTTGTAAGTGAGGCCACCGACAGT & 63 \\
\hline NT158 & ATTAGTACTGAGAAGTGTTTTGAACGGT & 56 \\
\hline NT159 & TTTCAGGAAAACAATATGGAAACCAGGCAAAGAATAAC & 61 \\
\hline NT160 & AAGTCACGCAGCCTTGCTCGCCATTCAGGCTCATAAAT & 68 \\
\hline NT161 & ATCGCAATACGTAGAAGTGTTGGGAAGGGCGTGGCGAA & 69 \\
\hline
\end{tabular}




\begin{tabular}{|c|c|c|}
\hline NT162 & AGAGAATCGTCTGAAGAA & 47 \\
\hline NT163 & CACACGATTTAAAGGGACCAGTAAAGCACGTGGCG & 66 \\
\hline NT164 & ACGCCAGGAGCTAAACAGGAGTCGTTAGGCGCTTAGCTGCGCGGGC & 74 \\
\hline NT165 & TGGCCAAAGAATACCAATATTCATTAAATTAACACATTTGACGGGGAAA & 64 \\
\hline NT166 & CCATACTGACCTGAAAGCGTACAGAGATAATGGATTACA & 62 \\
\hline NT167 & ATGGTTACAGAGTGGCGCTTTGACGTAAAAGGGATTCACGTAA & 67 \\
\hline NT168 & CGTCACCAGCACAGAGGAACGTGGCCTAAAG & 66 \\
\hline NT169 & GCTACAGATAACGTGCTTTCCGCCGATTTAGACAGTATA & 64 \\
\hline NT170 & TTTTACACCCGCCAATCAGAGCTTTT & 59 \\
\hline NT171 & CTTAGCGAACTGATAGCCACGC & 58 \\
\hline NT172 & AACAGTGCCCTAAAACATCGCTTTGAATGGCTATTAGTCTTT & 64 \\
\hline NT173 & AGTGTATAAAAGAAGAGCGGTCACATGCGCC & 63 \\
\hline NT174 & GCTAAGAAAGCGTAAAGCACTAAAAACCATCACCCAAACGAAAAA & 65 \\
\hline NT175 & TTTTCGGGCGCTAGTAACCACCTTTT & 61 \\
\hline NT176 & AGCAAATAAATATCAATCATT & 43 \\
\hline NT177 & GCCGGCGTGAGGCGGTCAGTAAATACCGAACGAACACT & 70 \\
\hline NT178 & TGCTTGGTCAGTTGGCAACTAAAAT & 58 \\
\hline NT179 & GGAGCCCGTTGAAAGGGCCCACGATGAATTGAGGATTGTTCC & 70 \\
\hline NT180 & TTTTGGGTCGAGGTGCCGAAAGGAGTTTT & 64 \\
\hline NT181 & TCAATTAACAACTAAGCAT & 44 \\
\hline NT182 & ATCTTTAAATAGATAATAGGGCGAGATACATTTG & 55 \\
\hline NT183 & CCGTCTAGTCCACTATTAAAGTTCCGAAATCGGCAGGTTTGCAGAC & 67 \\
\hline NT184 & TTTTAACGTCAAAGGGTCAAGTTTTTTGTTTT & 57 \\
\hline NT185 & AGCCGTCGGAGCACCAATATCGAACCTCGAAAAATCTAAAGCCGCCTGC & 71 \\
\hline NT186 & AGTTTGGTTATAAATCAAAAGTTGC & 51 \\
\hline NT187 & AGATCATGGGCTACGTGTCGGAACCGAGAAAGGAAGGGGGCA & 71 \\
\hline NT188 & GCCTTGAGTGAGGTTATATCAACACCGATTTAGAGCCACCT & 66 \\
\hline NT189 & TTTTGTTTGATGGTGGAACGTGGACTCCTTTT & 62 \\
\hline NT190 & CAATTAGAAGTATTAGCAATTC & 45 \\
\hline NT191 & AGCCCTTCACTATTAATGGAGCGGCTGA & 63 \\
\hline NT192 & GGGTAACATTGGAACAAAATGAATGGAAACCTGTCGTGGAA & 65 \\
\hline NT193 & TTTTGTTTTTCTTTTCACCAGTGCCCAGCAGGCGAAAATCCTTTTT & 67 \\
\hline NT194 & ATATTCGTATTAAATCTCCTGA & 46 \\
\hline NT195 & CGAACGTCGCCTGGCCACAAAACTTTCTGAGAGAGAATA & 66 \\
\hline NT196 & GTTTGAGCAACAGCTGATTGCAAGCGGTCCACGCTAAATCCCAACA & 70 \\
\hline NT197 & TTTTGGCGGTTTGCGTATTTTGCATCATTGGGCGCCAGGGTGTTTT & 70 \\
\hline NT198 & CGCTGATGATGGCAATTAGAA & 53 \\
\hline NT199 & TTGACTTCTGAATAATGTGCACGT & 55 \\
\hline NT200 & TTTTGCTCACTGCCCGCTTTACATTAA & 59 \\
\hline NT201 & CGGAACCATATCAAACAGT & 50 \\
\hline NT202 & ATTGAAGGGTTCATCAATATAATCAATTATCATCATATCTTTGCC & 60 \\
\hline
\end{tabular}




\begin{tabular}{lll}
\hline NT203 & AAAACAGACGTCAGATGAATACAAT & 53 \\
\hline NT204 & AAACCATTATTTTGGAGCTGCATTAGAAACCACCAGAATTTAAAA & 63 \\
\hline NT205 & ATTGCGGTGAGAATGATAGATTTTCCGCTCACAATTCCAATCATGG & 66 \\
\hline NT206 & CTCCCAGTCGCGGCCAACGCGCGGGGAGATTTT & 73 \\
\hline NT208 & TTCATTTTACATCGTGAAT & 71 \\
\hline NT209 & AACGGGAAATTGTTATCAGGTTTAAAAT & 44 \\
\hline NT210 & TGTGTATTCGCCTGAGAG & 54 \\
\hline NT211 & TCATAGCAAGCTTGACGTTGT & 51 \\
\hline NT212 & AGCTCGAGACTCTAGCCAGGG & 55 \\
\hline NT213 & GACTAAGTTACAAAATCGCGCATTGCTTGGAGAAATACAGTAAATT & 60 \\
\hline NT214 & GCGAATTAGCCAGTGCCTGTTTCC & 63 \\
\hline NT215 & GCAGGTCATTCGTACACAACAGGTGCCTCTAA & 61 \\
\hline NT216 & CGACGTTCATTTCAATTCATT & 64 \\
\hline NT217 & TTTTTTGGGTAACGAGGATCCCTTTT & 50 \\
\hline NT218 & TCAAGAACTGTAAATGGTGCCTACCG & 57 \\
\hline NT219 & TAATGTGAGTGCGCCATTGGTAATATCCAGAAACGCTC & 59 \\
\hline NT220 & TTACCTTAACAGTAGCGCAACAACTCAAACTATCGAATAAC & 65 \\
\hline NT221 & CTGCAAGCGCCAGCATCGGTGCATCACTTGCCTGATTCTTTG & 62 \\
\hline NT222 & CTTGCTTAACAAAATTAATTAACCTGAGCAAAAGAAGATGAT & 70 \\
\hline NT223 & CAATATACAATTTCATTTGAAAAAA & 60 \\
\hline NT224 & AGATGGATTTAGGGGATGTGTTTTCCCAGTCACGCATGCCT & 46 \\
\hline TTTTTCGCTATTAGCGATTAAGTTTT & 68 \\
\hline & & 52 \\
\hline
\end{tabular}

